\title{
Kulturelles Lernen an Texten russischer Migrationsliteratur im Lehramtsstudium
}

The present situation in our society places high demands of teachers of foreign languages concerning their cultural competences. The article considers the use of texts of Russian-speaking migration literature in the training of future teachers of RFL in the development of the cultural competence with the aim to describe arising difficulties and resources. First, the article introduces the current European concepts of cultural education (inter- and transcultural education, didactics of understanding others). Second, the article presents specific texts of migration literature for teaching RFL in higher education and justifies this choice. Third, the article describes an academic course, in which the texts were treated methodologically. In doing so, the article reveals the potential of migration literature for the cultural education of future teachers. In conclusion, it presents a small research study of the reflexion on cultural education by future teachers of L2 Russian.

Современная ситуация в обществе предъявляет к преподавателям иностранных языков высокие требования в области культурных компетенций. В статье рассматривается использование текстов русскоязычной миграционной литературы при подготовке будущих учителей РКИ с целью описать возникающие в связи с этим проблемы и возможности их решения. Во введении освещены актуальные европейские концепции обучения языку с учётом специфики межкультурного общения (кросскультурное и транскультурное обучение, дидактика понимания других). Далее приведены примеры текстов миграционной литературы для преподавания РКИ в ВУЗах и обоснован их выбор. В основной части

Anastasia Drackert und Katrin Bente Karl (Hg.), Didaktik der slawischen Sprachen

Beiträge zum 2. Arbeitskreis in Innsbruck (19.02.-20.02.2018)

C 2019 innsbruck university press, ISBN 978-3-903187-80-1, DOI 10.15203/3187-80-1 
представлен учебный курс, в котором эти тексты были обработаны методически. При этом показаны возможности использования миграционной литературы для подготовки будущих преподавателей в аспекте межкультурной коммуникации. Заключение знакомит с небольшим анализом отзывов участников этого курса, будущих преподавателей РКИ.

\section{Kulturelles Lernen}

Unter dem Dach des Begriffs kulturelles Lernen sind die Konzepte des interkulturellen Lernens, der Didaktik des Fremdverstehens und des transkulturellen Lernens verortet. Sie verfolgen das Ziel, den Wechsel zwischen Innen- und Außenperspektive zu veranlassen und ein Verständnis für kulturelle Alterität zu schaffen. Die Unterschiede zwischen den Konzepten des kulturellen Lernens beziehen sich auf die Definitionen des Kulturbegriffs, der für die jeweilige Ausrichtung des Konzepts ausschlaggebend ist.

So liegt dem im Gießener Graduiertenkolleg „Didaktik des Fremdverstehens" unter der Leitung von Lothar Bredella entwickelten Begriff des Fremdverstehens ein traditionelles Konzept voneinander abgrenzbarer Kulturen zugrunde (vgl. Bredella \& Christ 1995). Dieses Kulturmodell ist seit dem Ende des 18. Jahrhunderts durch Johann Gottfried Herder (1774, 1990) geprägt und stellt Kulturen als in sich geschlossene Kugeln dar. Auch interkulturelles Lernen basiert auf der Vorstellung von zwei voneinander getrennten Kulturen, die sich annähern und aneinander stoßen. Es handelt sich um die binäre Opposition des Eigenen und des Fremden und um einen Perspektivenwechsel, der die Sicht der Anderen verständlicher machen soll. Die Sensibilität für kulturelle Unterschiede soll dabei geschärft werden. Sowohl das Fremdverstehen als auch die interkulturelle Didaktik sondern das Fremde aus, mit dem Ziel, es besser zu verstehen. 
Somit bleiben diese zwei Konzepte des kulturellen Lernens der Dichotomie des Eigenen und des Fremden verhaftet.

Anstelle der Grenzziehung zwischen dem Eigenen und dem Fremden tritt im Kontext des kulturellen Lernens der durch die kulturelle Hybridität der Gesellschaft bedingte Begriff der Transkulturalität hervor. Im Lichte der Transkulturalität erscheint Kultur als hybrider, prozesshafter und diskursiver Begriff. Wolfgang Welsch widersetzt sich der traditionellen Auffassung von Kultur als einer homogenen Instanz und präsentiert einen veränderten Kulturbegriff, der sich auf stark miteinander verflochtene Kulturen bezieht (vgl. Welsch 2012: 146). Demnach sind Kulturen extern vernetzt und weisen einen internen Hybridcharakter auf: „Da die Heranwachsenden schon alltäglich mit einer weitaus größeren Anzahl kultureller Muster bekannt werden als dies in der Elterngeneration der Fall war, können sie bei ihrer kulturellen Identitätsbildung eine Vielzahl von Elementen unterschiedlicher Herkunft aufgreifen und verbinden. Das gilt nicht etwa nur für Migranten, sondern generell. Heutige Menschen sind zunehmend in sich transkulturell.“ (Welsch 2012: 149)

Dennoch steht ein kompletter Verzicht auf interkulturelles Lernen im Fremdsprachenunterricht nicht im Raum. Transkulturelles Lernen ergänzt ein friedliches Neben- und Miteinander von Kulturen und entwickelt das Paradigma kulturellen Lernens entsprechend den Anforderungen der globalisierten und hybridisierten Gesellschaft weiter (vgl. Delanoy 2014: 26). Dabei geht es um Transformationen interkulturellen Lernens ,im Lichte transkultureller Positionen“ (Delanoy 2014: 32), um die Verstehenserweiterung ohne klare Abgrenzung von Eigen und Fremd.

Kulturelles Lernen erschließt den Fremdsprachenunterricht durch die Entstehung einer dritten Kultur (Kramsch 1993), einen kulturellen Zwischenraum, der die Kulturen der Lernenden und die Kultur der Zielsprache einander öffnet und die Grenzen zwischen Kulturen aufbricht. So wird der Fremdsprachenunterricht zu einem Ort, an dem Mehrdeutigkeiten und 
Veränderungen, Überlappungen, Vielfalt und Brüche von eindeutigen Dichotomien zugelassen sind. Der Fremdsprachenunterricht wird zu einem Aushandlungsraum, in dem Fragestellungen außerhalb der Dichotomie des Eigenen und des Fremden lokalisierbar sind. Zahlreiche Konzeptualisierungen kulturellen Lernens (Hallet 2002; Fäcke 2006; Caspari \& Küster 2010) im Fremdsprachenunterricht weisen auf eine besondere Relevanz des Entstehens des „Gemeinsamen“ (vgl. Küster 2010: 41) hin.

\section{Kulturelle Kompetenzen in der Lehramtsausbildung der Fremdsprachen- lehrerInnen}

Die Lehramtsausbildung für FremdsprachenlehrerInnen sieht eine Kombination aus Fachwissenschaften, Fachdidaktik und Sprach- und Schulpraxis vor. Der Erwerb deklarativen Wissens, prozeduraler Fertigkeiten und persönlichkeitsbezogener Kompetenzen im Studium geht auf den Kompetenzbegriff im GERS zurück, der sowohl allgemeine als auch kommunikative Sprachkompetenzen umfasst. Eine Verknüpfung bereichsspezifischer Kenntnisse, Fertigkeiten, Strategien und persönlicher Einstellungen hinsichtlich kultureller Thematik spielt dabei eine tragende Rolle (vgl. o.A. 2016).

Kulturelle Kompetenzen setzen sich aus interkulturellen, interkulturellkommunikativen und transkulturellen Kompetenzen zusammen. Im Rahmen der Kompetenzorientierung des Fremdsprachenunterrichts seit dem Beginn des 21. Jahrhunderts tritt das Modell von Byram (1997) zur Vermittlung interkultureller kommunikativer Kompetenz in den Vordergrund. Dabei wird zwischen der interkulturellen Kompetenz und der interkulturellen kommunikativen Kompetenz unterschieden. Während die interkulturelle Kompetenz in der Muttersprache zum Vorschein kommt (vgl. Byram 1997: $70 \mathrm{ff}$ ), steht ein adäquater Gebrauch der jeweiligen Zielsprache im Fokus der interkulturellen kommunikativen Kompetenz. 
Die interkulturelle Kompetenz umfasst kognitive, affektive und evaluative Teilkompetenzen (vgl. Bredella 2017: 150) und bildet einen Teil der sozialen Kompetenz. Ein wichtiges Ziel beim Aufbau der interkulturellen Kompetenz ist es, aufzuzeigen, dass die Übernahme kultureller Deutungsmuster aus einer Kultur in eine andere kulturelle Umgebung problematisch ist. Unter kulturellen Deutungsmustern wird ein ,im Sozialisationsprozess erworbenes Wissen über die Zeit, das uns in der Alltagskommunikation und im Alltagshandeln Orientierung gibt und so etwas wie eine gemeinsame Wirklichkeit schafft" (Altmayer 2009: 128) verstanden. Kompetentes interkulturelles Handeln zeigt sich insofern in einer erfolgreichen Bewältigung zwischenkultureller Situationen, als eigene Werte relativiert werden.

Die interkulturelle kommunikative Kompetenz lässt sich als eine Kombination kognitiver (kulturelles und landeskundliches Wissen), affektiver (Bereitschaft, die Perspektive zu wechseln) und handlungsbezogener Teilfertigkeiten (Interpretations- und Lernfähigkeit) beschreiben (vgl. Byram 1997: 34). Für den fremdsprachlichen Unterricht von LehramtsanwärterInnen ist es von besonderer Bedeutung auch fremdsprachlich-kommunikative, soziolinguistische und diskursive Teilkompetenzen zu fördern, da sie einen wesentlichen Beitrag zur interkulturellen kommunikativen Kompetenz leisten und sich unmittelbar auf den angemessenen Sprachgebrauch in einer Zielsprache beziehen. Der Aufbau und die Unterstützung interkultureller sprachlicher Beziehungen zwischen Menschen unterschiedlicher Kulturen verstehen sich als grundlegende Elemente der interkulturellen kommunikativen Kompetenz. Unter den Fördermaßnahmen im Kontext lehramtlicher philologischer Studien ist die Arbeit mit literarischen Texten besonders hervorzuheben (vgl. Bredella 2017: 234; Freitag-Hild 2017: 149).

Eine weiterführende Ergänzung zu den erwähnten kulturellen Kompetenzen hinsichtlich der Identitätsentwicklung der Lernenden vor dem transkulturellen gesellschaftlichen Hintergrund bieten transkulturelle Kompetenzen. Insbesondere Identitätskompetenz und Bewusstsein für Alterität

Anastasia Drackert und Katrin Bente Karl (Hg.), Didaktik der slawischen Sprachen

Beiträge zum 2. Arbeitskreis in Innsbruck (19.02.-20.02.2018)

(C) 2019 innsbruck university press, ISBN 978-3-903187-80-1, DOI 10.15203/3187-80-1 
(vgl. Alter 2016: 57), die sich auf die Selbstfindung des Individuums und unterschiedliche Ausprägungen des Andersseins beziehen, können als zeitgerechte zielangebende Richtungen des Fremdsprachenunterrichts beschrieben werden. Der Begriff der Identitätskompetenz bezeichnet einen bewussten Umgang mit Repräsentationen von Anderssein, eine kritische Reflexion der eigenen Identität und respektvollen Kontakt mit dem Anderen (ebd.). Bewusstsein für Alterität soll eine oberflächliche und vereinfachte Wahrnehmung des Anderen verhindern und bedeutet, ,, dass der Andere in seinem Anderssein verbleiben kann" (ebd.: 58). Auch diesen Teilkompetenzen kommt in der LehrerInnenbildung in der gegenwärtigen Gesellschaft eine tragende Rolle zu, denn die Schule stellt einen Abdruck der modernen Gesellschaft in all ihrer Komplexität, mit unterschiedlichen Formen von Anderssein und kultureller Hybridisierung dar.

Im Rahmen der Lehramtsausbildung zukünftiger FremdsprachenlehrerInnen spielen literarische Texte eine besondere Rolle. Die Verbindung zwischen dem Aufbau kultureller Kompetenzen im Kontext kulturellen Lernens und dem literaturbezogenen Fremdsprachenunterricht steht im Fokus mehrerer Forschungsarbeiten (Hallet 2002; Fäcke 2006; Freitag-Hild 2010; Alter 2016; Ivanova 2016). Diese zeigen, dass literarische Texte zur Förderung kultureller Kompetenzen erfolgreich herangezogen werden können.

\section{Textauswahl für kulturelles Lernen}

Transkulturelles Lernen bringt transkulturelle Aspekte der Gesellschaft ans Licht und verlangt nach einer veränderten Textauswahl für den Fremdsprachenunterricht (vgl. Alter 2016: 52). Die Identitäten literarischer Figuren als hybride kulturelle Ausprägungen beeinflussen die Textauswahl. 
Der Paradigmenwechsel des kulturellen Lernens zieht den Übergang von inter- zu transkultureller Literatur nach sich. Der Unterschied zwischen den beiden liegt in der Auffassung der Identitätskonstruktionen der Protagonisten. Ethnisches Anderssein spielt in der transkulturellen Literatur eine mindere Rolle, es geht primär um die diversen Formen von Alterität. Grit Alter beschreibt treffend die Merkmale transkultureller Literatur: ,(Kulturelle) Identität ist nicht Problem, sondern Faktor und spielt in der Entwicklung der Narration trotzdem eine tragende Rolle - dies jedoch mit einem ergänzenden und elementaren Verständnis als sinnstiftend, kraftgebend und mensch-definierend, nicht problem-definierend. Die Romanfiguren sind nicht das Problem, das gelöst werden muss, sondern werden zu aktiven und selbstbestimmten Problemlösenden. Sie sind aktiv Handelnde, die zum Ausgang der Erzählung durch Kreativität, Energie und Idealismus beitragen, sie sind dabei nicht einseitig positiv dargestellt, sondern aufgeklärt ausgewogen.“ (Alter 2016: 54)

Genauso wie das transkulturelle Lernen das interkulturelle Lernen nicht vollkommen ablösen kann, ist es fast unmöglich eine deutliche Grenze zwischen inter- und transkultureller Literatur zu ziehen. Alter (ebd.: 55) schlägt ein Kontinuum vom inter- zum transkulturellen Lernen vor (Tab. 1). 


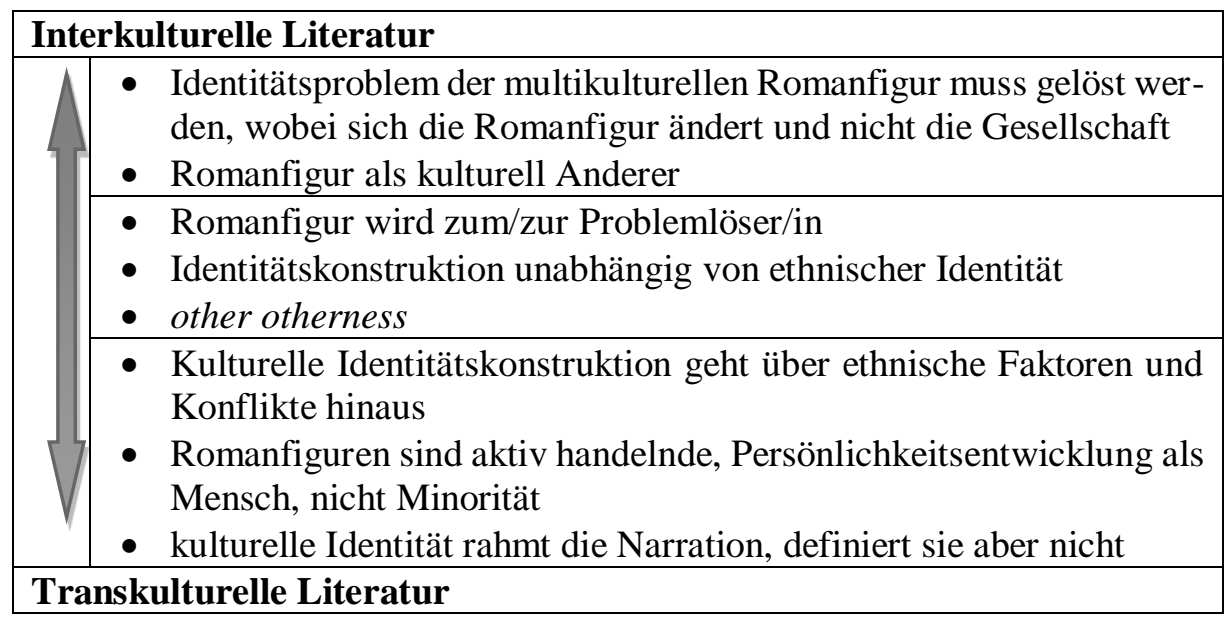

Tab. 1 Kontinuum vom inter- zum transkulturellen Lernen (Alter 2016: 55)

Es zeigt sich, dass die Fokussierung auf die Identität der Protagonisten entscheidende Merkmale zur Bestimmung der inter- oder transkulturellen Ausrichtung der Literatur liefert. Runde, komplexe Charaktere, die als selbstbewusste und aktiv handelnde Personen trotz unterschiedlich ausgeprägter Alterität auftreten, kennzeichnen transkulturelle Literatur.

In diesem Zusammenhang wird deutlich, dass Migrationsliteratur transkulturell bestimmt ist und sich für transkulturelles Lernen bestens eignet. Migrationsliteratur setzt sich mit der Thematik der kulturellen Alterität, interkulturellen Begegnungen sowie mit Versuchen, die eigene Identität besser zu verstehen bzw. zu definieren auseinander. Darüber hinaus stehen auch allgemeine Themen wie Normen und Werte, Liebe, Familie, Pubertät, Gender, Arbeit sowie soziale und gesellschaftliche Bedingungen im Fokus der Migrationsliteratur. Britta Freitag-Hild (2010) untersucht den Einsatz der Migrationsliteratur aus der Perspektive der inter- und transkulturellen Literaturdidaktik des Englischen und bestätigt das didaktische Potenzial dieser literarischen Texte aufgrund der folgenden Thesen: 
Migrationsliteratur bietet vielfältige Identifikationspotenziale für Lernende, fördert die Fähigkeit zur Perspektivenübernahme und zur Aushandlung verschiedener Sichtweisen, veranlasst Lernende zur Auseinandersetzung mit fremdsprachigen Diskursen und bricht verfestigte kulturelle Deutungsmuster auf (vgl. Freitag-Hild 2010: 8). Darüber hinaus trägt FreitagHild zur Erweiterung des Literaturkanons im Englischunterricht durch die Literatur der British fictions of migration bei. Trotz der Gefahr einer Reduzierung der Thematik bzw. einer einschränkenden Fokussierung auf den Migrationshintergrund der Protagonisten (vgl. Alter 2016: 52) bietet Migrationsliteratur eine breite Palette an konstruktiven Fragestellungen im Kontext des kulturellen Lernens.

\subsection{Warum eignet sich Migrationsliteratur für den Einsatz im kultu- rellen Lernen der LehramtsanwärterInnen?}

Migrationsliteratur ist ein Narrativ, das eine künstlerische Aufarbeitung unterschiedlicher geschichtlicher Ereignisse emotional und personalisiert bietet. Es geht um die Geschichte der Menschheit, in dem konkreten Fall um eine bildliche Darstellung der russischen Migrationsgeschichte durch das Prisma des literarischen Erzählens. Literatur bildet die Realität nicht einfach ab, dennoch können literarische Texte im Lichte des New Historicism mit Berücksichtigung des gesellschaftlich-politischen Kontextes gelesen werden (vgl. Kaes 1990: 64). Im Sinne des wide reading nach Wolfgang Hallet, wenn literarische Texte auf die Spuren gesellschaftlicher Diskurse geprüft und verstanden werden (vgl. Hallet 2007: 44), lassen sich Texte russischer Migrationsliteratur zu verschiedenen Aspekten des kulturellen Lernens in Beziehung setzen. Dabei spielt die historische Komponente der Texte eine wichtige Rolle für die Kontextualisierung der russischen Geschichte und Literatur.

Anastasia Drackert und Katrin Bente Karl (Hg.), Didaktik der slawischen Sprachen

Beiträge zum 2. Arbeitskreis in Innsbruck (19.02.-20.02.2018)

(C) 2019 innsbruck university press, ISBN 978-3-903187-80-1, DOI 10.15203/3187-80-1 
Migrationsliteratur erlaubt es, kognitivistische Ansätze von kulturellen Kompetenzen mit Emotionen, d.h. affektiven Komponenten, zu verbinden. Hinsichtlich des Einsatzes im Fremdsprachenunterricht geht es um das Zusammenschließen zweier Modi des Denkens nach Jerome Bruner, des narrativen und des paradigmatischen Modus, die konkrete Wege zeigen, Erfahrungen zu ordnen und die Realität zu konstruieren (vgl. Bruner 1986: 11). Migrationsliteratur stellt ein besonders geeignetes Beispiel dar, das den narrativen Modus des Denkens, der durch Unbestimmtheit, Unsicherheit, Anschlussmöglichkeit und Emotionen gekennzeichnet wird und über das Faktische hinausgeht, mit dem paradigmatischen Denken, welches das faktuale Wissen, das das Geschichtliche und Landeskundliche betrifft, verbindet.

Ein weiteres Argument für den Einsatz russischer Migrationsliteratur in der Ausbildung angehender FremdsprachenlehrerInnen ist ein besonderer Umgang mit Sprache(n). Ästhetisch-literarische Aspekte der Migrationsliteratur erlauben in den Texten eine Metaperspektive zu eröffnen, da sie sich nicht auf die Welt beziehen, sondern ,Sichtweisen der Welt präsentieren“"(Küster 2015: 22). Nicht nur die Gefühle, die Ereignisse, die in den Texten vorkommen, sondern vor allem die Sprache setzt solche Kategorien in Beziehung wie damals und jetzt, oder hier und dort. Die Sprache greift die für die AutorInnen spezifischen Diskurse auf und reflektiert somit die Flexibilität und Hybridität menschlicher Denkweisen und Kultur. Genauso spannend ist das Analysieren der Migrationstexte in Bezug auf literarische Verfahren, die das Fremde, das Vertraute und das neue Eigene wiedergeben. Im Sinne der sprachlich-kommunikativen Ausrichtung des kulturellen Lernens und der gegenseitigen Bereicherung literarischer und kultureller Lernprozesse im Studium (vgl. ebd. 25) sollen diese Aspekte nicht außer Acht gelassen werden.

Der Zusammenhang zwischen der Geschichte, ästhetischen Aspekten sowie der emotionalen Aufladung der Texte spricht für den Einsatz der 
Migrationsliteratur im Kontext des kulturellen Lernens im Fremdsprachenunterricht. Küster betont, dass „Verarbeitungstiefe und Nachhaltigkeit des Lernens dann am intensivsten ausgeprägt sind, wenn Kognition und Emotion gleichermaßen beteiligt sind und das Lernen als bedeutsam für eigene Sinnkonstruktionen wahrgenommen wird, d.h. wenn Lernen zu einer persönlichen Erfahrung wird“" (Küster 2010: 42). Im Folgenden wird auf die Texte russischer Migrationsliteratur eingegangen, die sich für kulturelles Lernen im Lehramtsstudium eignen.

\subsection{Texte russischer Migrationsliteratur für kulturelles Lernen}

Der Begriff der Migrationsliteratur wird relativ breit gefasst. Die literaturwissenschaftliche Perspektive bietet unterschiedliche Zugänge zur Bestimmung dieser Gattung, wie beispielsweise Produktions- oder Rezeptionsparameter, thematische bzw. inhaltliche Kriterien und auch künstlerische Verfahren (vgl. Hausbacher 2009: 25ff). Dies führt zu einem breiten Spektrum der Bezeichnungen, die unter dem Oberbegriff der Migrationsliteratur verortet werden: Exil- und Migrantenliteratur, neue Weltliteratur, interkulturelle/interlinguale Literatur, transkulturelle Literatur. Im russischen Kulturkontext des 20. Jahrhunderts dominiert in diesem Zusammenhang der Begriff der Emigrationsliteratur, der sich auf kritische Auseinandersetzungen von Autoren und Autorinnen mit dem sozialistischen Staat stützt bzw. politisch motiviert ist (vgl. Hausbacher 2009: 31).

Die russische Migration im 20. Jahrhundert gliedert sich in vier Wellen (vgl. Hausbacher 2009: 32), die von politischen bzw. gesellschaftlichen Ereignissen ausgelöst wurden. Die erste Welle beginnt direkt nach der Oktoberrevolution 1917 und dauert während des Bürgerkriegs an. In dieser Zeit des Umbruchs verlassen viele Intellektuelle und Literaten das Land.

Anastasia Drackert und Katrin Bente Karl (Hg.), Didaktik der slawischen Sprachen

Beiträge zum 2. Arbeitskreis in Innsbruck (19.02.-20.02.2018)

(C) 2019 innsbruck university press, ISBN 978-3-903187-80-1, DOI 10.15203/3187-80-1 
Die zweite Welle verläuft während des Zweiten Weltkriegs. Es sind diejenigen BürgerInnen der Sowjetunion, die unabhängig von der Art ihres Kontaktes zu den Westmächten mit einer Strafverfolgung nach dem Krieg rechnen mussten. Die dritte Welle umfasst eine längere Periode, vom Ende der 1960er Jahre bis zum Zerfall der Sowjetunion. In dieser Zeit kommt es sowohl zu politisch motivierten Ausweisungen von Dissidenten als auch zu einer Massenemigration sowjetischer Juden. Die vierte Welle findet in den 1990er Jahren statt, sie ist weniger politisch motiviert. Innere Probleme Russlands in der Kombination mit der Öffnung der Grenzen sind die Auslöser dieser Emigrationswelle. Diese Gliederung zeigt deutlich, dass die Geschichte der Migration die Geschichte des Landes reflektiert. Die literarische Aufarbeitung der Ereignisse, die sich zum Teil in Texten der Migrationsliteratur äußert, begünstigt das empathische Miterleben der Geschichte bei der Rezeption.

Die folgende Textauswahl ist durch die historische Prägung des Erzählten sowie eine spannende Breite der Identitätskonstruktionen der Protagonisten bedingt. Dank der kulturellen Vielstimmigkeit der Texte, die eine eindeutige Leseperspektive nicht unterstützt, wird kulturelles Lernen initiiert bzw. gefördert.

Die Erzählung „V Pariže“ (dt. Übersetzung In Paris) des Nobelpreisträgers für Literatur Ivan Bunin zeigt Szenen aus dem Leben russischer EmigrantInnen in Paris nach der Oktoberrevolution 1917. Eine kurze Liebesgeschichte eines russischen Offiziers und einer russischen Dame offenbart die ganze Tragik des Daseins im fremden Paris. Stellvertretend für viele durch die Revolution gebrochenen Schicksale werden die Gefühle der gutsituierten bzw. adeligen Russen gezeigt, die in der Emigration ihr Leben neu beginnen müssen und um die Vergangenheit trauern. Dennoch erscheint das Licht am Ende des dunklen Tunnels in Gestalt der Liebe, die die Protagonisten zueinander finden. Ein spannender Sprachgebrauch veranschaulicht die Verbindung zur russischen Vergangenheit und der französischen Gegenwart. 
Der vierte Roman des Nobelpreisträgers Vladimir Nabokov „Pnin“(Pnin) zeigt Ausschnitte aus dem Leben eines Emigranten. Der Collegeprofessor Timofej Pnin unterrichtet in den 1950er Jahren in den USA russische Sprache und Literatur. Pnin ist ein Antiheld, seine Versuche, sich an das neue Leben und die gegenwärtige Umgebung anzupassen, scheitern meistens, dennoch stellt dieser Roman ein besonderes Beispiel der Migrationsliteratur dar. Der Protagonist ist eine komplexe, runde Figur, die menschliche Schwächen und Ängste sowie Ambitionen und Pragmatismus liebevoll spiegelt.

Im Zentrum von Vladimir Vertlibs Roman „Ostanovki v puti“ (Zwischenstationen) befindet sich eine russisch-jüdische Familie, die auf der Suche nach Freiheit viele Länder bereist. Die Handlung findet Ende der 1970er, Anfang der 1980er Jahre statt. Mehrmals landet die Familie in Wien, wo der Sohn ein Gymnasium besucht. Die unterschiedlichen zum Teil altersund erfahrungsbedingten Sichtweisen der Familienmitglieder äußern sich in alltäglichen Situationen, die zugleich tragische und komische Seiten des Lebens abbilden. Das stetig anwesende Gefühl der Fremdheit stiftet Unruhe in den Handlungen der Eltern und des pubertierenden Sohnes. Das Thema des Erwachsenwerdens verflicht die Narration. Die Situationsabhängigkeit und kontextuelle Bedingtheit der Sprache der Familienmitglieder stellt ein spannendes Untersuchungsobjekt dar, das die enge Verbindung zwischen der Kultur und dem Sprachgebrauch verdeutlicht.

Julija Kisinas Roman „Vesna na lune“ (Frühling auf dem Mond) präsentiert Kindheitserinnerungen an das Leben in der Sowjetunion der 1970er Jahre aus der Perspektive einer erwachsenen Emigrantin. Die Kindheit der Protagonistin an der Schwelle zum Erwachsenwerden erlaubt keine deutlichen Antworten auf die ewigen Fragen der Liebe, des Todes und des Lebens. Viel Komik sowie Alltagssatire in Kombination mit Nostalgie nach der zauberhaften Zeit der Kindheit zeichnen diesen Roman aus. 
Ein besonderes Beispiel der Migrationsliteratur bietet die Novelle Viktorija Tokarevas „Svoja pravda“ (Eine Liebe fürs ganze Leben). Die Protagonistin Marina erlebt die Binnenmigration, als sie nach dem Zerfall der Sowjetunion aus Aserbaidschan nach Moskau umziehen muss. Der Perspektiven- und Stellenwechsel der russischen Frau spiegelt die Verhältnisse in der Zeit des Umbruchs, die Tragik der Situation wird durch die unglückliche Liebe hervorgehoben.

Dina Rubinas Monolog „Vyveska“(Ein Aushängeschild) vertont die Sicht einer Israelitin auf ihr Leben im gelobten Land in den 1990er Jahren durch das Prisma ihrer russischen bzw. sowjetischen Vergangenheit. Die Kurzerzählung erinnert an alltägliche Gespräche zwischen guten Bekannten, mit einem großen Anteil an Anekdoten und Beschreibungen, zugleich wird aber die Verflochtenheit des menschlichen Denkens deutlich, das sich durch das unmögliche Teilen von Gegenwart und Vergangenheit zeigt.

Die Erzählsammlung „Russendisko“ (Russendisko) Vladimir Kaminers präsentiert humorvolle Reflexionen und Geschichten aus dem Leben eines jungen russischen Emigranten im Berlin der 1990er Jahre. Mit Leichtigkeit spricht der Protagonist über seine Erfahrungen und Herausforderungen in der deutschen Hauptstadt, sowie über Begegnungen und den kulturellen Austausch mit ihren BewohnerInnen. Nicht zu knapp nehmen auch Liebe und Freundschaft ihren Platz in den Erzählungen ein.

All diese Texte bringen unterschiedliche Perspektiven ans Licht und verlangen nach einer Relativierung der russischen Geschichte. Sie vertreten alle Wellen der russischen Migration und ermöglichen ihre Einbettung in die Weltgeschichte durch die Schicksale der Hauptcharaktere. Die Texte wurden in einer Lehrveranstaltung behandelt, die im Weiteren beschrieben wird. 


\section{Die Lehrveranstaltung „GrenzgängerInnen: Migrations- literatur und ihr Einsatz im Russischunterricht" als Prozess- initiierung im Hochschulunterricht}

Ein bildender und zugleich kompetenzentwickelnder Hochschulunterricht muss auch im Hinblick auf drei Arten von Prozessen initiierend wirken, nämlich auf kognitive, diskursive und interaktionale Prozesse (vgl. Hallet 2013: 19). Das bedeutet, dass Studierende Wissen in einem konkreten Wissensgebiet besitzen, dass sie fachliche und außerfachliche Kommunikation über das zu lernende Thema kennen und daran teilnehmen können, und dass sie an der Aushandlung der Bedeutungen aktiv partizipieren (vgl. ebd.). Diese Punkte sind für die Lehrveranstaltung „GrenzgängerInnen: Migrationsliteratur und ihr Einsatz im Russischunterricht" ausschlaggebend gewesen.

Das Lehramtscurriculum des Fachbereichs Slawistik der Universität Salzburg sieht ein Proseminar zum Thema „Historische Aspekte der russischen Literatur und Kultur aus fachdidaktischer Perspektive" vor. Im Rahmen dieser Lehrveranstaltung sollen Studierende Querverbindungen zwischen Fachinhalten, fachdidaktischen Anliegen und der Schulpraxis herstellen und fächerübergreifend agieren. Im Wintersemester 2017 lautete der Titel des Kurses „GrenzgängerInnen: Migrationsliteratur und ihr Einsatz im Russischunterricht". Die Lehrveranstaltung beschäftigte sich mit den oben beschriebenen Texten russischer Migrationsliteratur. Auf der Basis von theoretischen Texten wurden zunächst kulturelle Entwicklungen der russischen Migration und ihre Bedeutung im literarischen Text erarbeitet. Dann wurden die Studierenden mit den theoretischen Grundlagen des kulturellen Lernens und praktischen Beispielen von Methoden für den inter-/transkulturellen Literaturunterricht vertraut gemacht. In einem weiteren Schritt wurden literarische Texte von den Studierenden analysiert und für den Einsatz im Russischunterricht fachdidaktisch aufbereitet. 
In Anlehnung an das Lehrbuch „Grenzgängerinnen: Migrationsgeschichten in der Gegenwartsliteratur" (Csorba et al. 2018), das im Rahmen einer Zusammenarbeit zwischen der Universität Salzburg und der Universität Debrecen publiziert wurde, wurden die Aufgabenstellungen für die Studierenden vorbereitet. Sie sollten zu den Texten bzw. Textauszügen Fragen und Übungen vorbereiten, die sich in drei große Gruppen gliedern, und zwar Aufgaben vor dem Lesen, während des Lesens und nach dem Lesen. Außerdem sollten die Studierenden sprachdidaktische Aufgaben erstellen, die darauf abzielen, literarische Texte für den Spracherwerb nutzbar zu machen. Einen der Schwerpunkte bildete das kulturelle Lernen, sodass Studierende beim Aufbereiten der Texte spezielle Aufgaben hinsichtlich der Inter- und Transkulturalität thematisieren sollten. Damit sollte ein Produkt entstehen, das fachspezifische Kenntnisse der Literaturwissenschaft (literaturwissenschaftliche Textanalyse) und fachdidaktisches Wissen (Leseaufgaben und Übungsaufgaben zur Entwicklung von Sprechfertigkeit) vereint und zugleich kulturelle Lernprozesse initiiert.

Die Aufgaben für Studierende fokussierten auf das Zusammenwirken von fachlichen und didaktischen Inhalten durch das Prisma des handelnden Subjekts. Die Aufbereitung der ausgesuchten Texte durch die Studierenden wurde als Auslöser für mentale Prozesse betrachtet, dabei ging es um die Kognitionen und Emotionen von Lehramtsstudierenden sowie um ihre Handlungsfähigkeit als Lehrkraft für Russisch. Die intendierten Lernergebnisse der Lehrveranstaltung wurden wie folgt formuliert: Die Studierenden

- können die Phasen der kulturhistorischen Entwicklung der russischen Migration aufzählen,

- sind in der Lage Grundbegriffe und Methoden der Textanalyse anzuwenden,

- können Texte der Migrationsliteratur fachdidaktisch aufbereiten und deren Einsatz im Unterricht planen, 
- können Unterrichtssimulationen unter Einbeziehung von Theorien kulturellen Lernens (inter- und transkulturelles Lernen, Didaktik des Fremdverstehens) gestalten.

Das allgemeine Ziel dieser Lehrveranstaltung bestand darin, Lernbedingungen zu schaffen, die den Studierenden die Bedeutung der Verbindung zwischen der Fachwissenschaft und der Fachdidaktik verdeutlichen und veranschaulichen. Die kompetenzorientierten Lernergebnisse befinden sich auf den folgenden Ebenen der Bloomschen Taxonomie der Lernziele: Wissen, Anwendung, Analyse und Synthese (Bloom et al. 1986). Eine solche Kompetenzorientierung erfolgt nach dem fachdidaktischen Prinzip des handlungsorientierten Lernens, das Theorie und Praxis literaturwissenschaftlicher sowie literatur-/und fachdidaktischer Diskurse vereint. Am Ende sollten die Studierenden ein Produkt vorstellen, das aus der Verknüpfung literaturwissenschaftlicher und fachdidaktischer Kenntnisse und Fertigkeiten resultiert, die sich Studierende im Laufe ihres Studiums angeeignet haben. Als Ergebnis sollten die Studierenden nach einem vorgegebenen Modell ihre eigenen Unterrichtsbeispiele für die Erarbeitung literarischer Texte im kulturellen Kontext des Fremdsprachenunterrichts erstellen und präsentieren. Die Auswahl der literarischen Texte bedingte die Verortung der Arbeit im Kontext des kulturellen Lernens. In diesem Zusammenhang wurden auch die folgenden Ziele gesetzt, die sich der Identitätskompetenz zuordnen: Die Studierenden können

- vermittelte Repräsentationen von Alterität hinterfragen,

- die eigene Identität und das Selbst reflektieren

- und ein Bewusstsein für kulturelle Ereignisse, Praktiken und Informationen entwickeln.

Während sich die intendierten Lernergebnisse im Bereich der Kognition und der Handlungsfähigkeit als Lehrkraft in einem konkreten Ausmaß relativ leicht überprüfen lassen, ist das Erreichen der Ziele der transkulturellen Kompetenz nicht eindeutig feststellbar. Das deklarative Wissen auf

Anastasia Drackert und Katrin Bente Karl (Hg.), Didaktik der slawischen Sprachen

Beiträge zum 2. Arbeitskreis in Innsbruck (19.02.-20.02.2018)

(C) 2019 innsbruck university press, ISBN 978-3-903187-80-1, DOI 10.15203/3187-80-1 
dem Gebiet der russischen Migration sowie das prozedurale Wissen der literarischen Textanalyse und der fachdidaktischen Aufbereitung des Materials wurde in den von den Studierenden vorbereiteten Lehrunterlagen, Unterrichtsplanungen und Simulationen sowie in einer kleinen schriftlichen Klausur sichtbar. Die Überprüfbarkeit der inter-/transkulturellen Ziele stellt eine größere Herausforderung dar, da kulturelle Kompetenzen schwer messbar sind (vgl. Frederking 2008; Byram \& Hu 2009). Byram stellt fest, dass sich interkulturelle Ziele nicht unbedingt als beobachtbares und messbares Verhalten oder Verhaltensänderungen formulieren lassen (vgl. Byram 1997: 49). Dennoch besteht ein Bestreben, Änderungen in der vorgegebenen Richtung wenigstens bemerkbar zu machen. Als eine mögliche Lösung des Dilemmas werden Portfolios zur Selbsteinschätzung vorgeschlagen (vgl. Schulz 2007; Byram \& Hu 2009). Sie sollen LernerInnen helfen, eigene Erfahrungen und Begegnungen mit Alterität zu analysieren und zu reflektieren. In unserem Fall wurde eine Reflexion der kulturellen Ziele im Rahmen einer Interviewstudie durchgeführt, die parallel zu der Lehrveranstaltung „GrenzgängerInnen“ abgehalten wurde.

\section{Die Interviewstudie „Was Lehramtsstudierende über den Einsatz russischer Migrationsliteratur in kulturellem Lernen denken"}

\subsection{Forschungsziele und Design}

Die Studie beschäftigt sich mit den Prozessen und Zwischenergebnissen des kulturellen und fremdsprachlich ausgerichteten Literaturunterrichts angehender RussischlehrerInnen. Die Studie verfolgt mehrere Ziele, vor allem aber sollte sie den Lernprozess reflektieren. Zum einen sollte die Studie kulturelles Lernen an Texten russischer Migrationsliteratur aus der 
Sicht der Studierenden wiedergeben, zum anderen sollten die Reflexionen der Studierenden als Ausgangspunkt zur Optimierung der Lehrveranstaltung dienen. Im Rahmen dieses Beitrags wird der Fokus auf die subjektive Sichtweise der Studierenden gelegt, die deren Annahmen, Wahrnehmungen, Kognitionen und Konzepte individuellen und kooperativen kulturellen Sprachenlernens unter den Bedingungen der Lehramtsausbildung spiegelt. Als Leitsatz gilt: „Die subjektive Dimension allen Verstehens, Erkennens und Erklärens muss daher auch in der Lehre zum expliziten, bewussten Ausgangspunkt möglicher Zugänge zu den Gegenständen werden und am Beginn einer Schrittfolge hin zu intersubjektiver Aushandlung und wissenschaft licher Fundierung der Erkenntnisse stehen" (Hallet 2013: 18). Primär geht es in der Studie nicht um die Textauswahl, sondern um die Bedeutungen, die bei den Studierenden entstehen. Somit soll die Untersuchung einen Beitrag dazu leisten, die subjektiven Ansichten der Studierenden in Bezug auf kulturelles Lernen und Migrationsliteratur sichtbar zu machen. Dabei sollte die Breite der subjektiven Bedeutungen aufgezeigt werden.

Die Studie sollte folgende Fragen beantworten: Inwiefern beeinflussen die Lebenserfahrungen der Lernenden ihre Einstellung zum kulturellen Lernen? Welchen Platz nimmt Migrationsliteratur im kulturellen Lernen aus der Sicht der Studierenden ein? Welche Aspekte des kulturellen Lernens an Texten russischer Migrationsliteratur werden von Studierenden für den Fremdsprachenunterricht als bedeutsam empfunden?

Um die subjektive Auffassung des kulturellen Lernens festzuhalten, wurde eine mündliche Form der Befragung, konkret ein qualitatives Interview, gewählt. Qualitative Interviews bringen die Befragten dazu, ,selbst Auskünfte darüber zu geben, was und warum etwas für sie relevant ist. [...] Für qualitative Interviews gilt das Prinzip der Offenheit; den Untersuchungsteilnehmern wird Raum für Elaborationen, Klarstellungen und Erklärungen und damit die Option zu tiefgründigeren und glaubwürdigeren Aus-

Anastasia Drackert und Katrin Bente Karl (Hg.), Didaktik der slawischen Sprachen

Beiträge zum 2. Arbeitskreis in Innsbruck (19.02.-20.02.2018)

(C) 2019 innsbruck university press, ISBN 978-3-903187-80-1, DOI 10.15203/3187-80-1 
künften gegeben.“(Riemer 2016: 162) Die freiwilligen StudienteilnehmerInnen (insgesamt 7) wurden in der Zeit kurz nach ihren Unterrichtssimulationen einzeln zum Interview eingeladen. Die Dauer des Interviews betrug ca. 25 Minuten.

Das Interview war halbstrukturiert-leitfadenorientiert aufgebaut, d.h., dass ein „Kompromiss zwischen z.T. vorgegebenen Fragen und dem Erzählenlassen“ durch „Leitfadenkomplexe [...], die den Themenschwerpunkten entsprechen" (Bock 1991: 94) zusammen mit den Befragten gesucht wird. Die Fragen wurden auf der Basis der Forschungsfragen vorformuliert, sodass zwei Themenkomplexe gebildet wurden. Die Aufwärmphase als Einstieg in das Gespräch umfasste Fragen, die einen unmittelbaren Bezug zum Studienalltag bzw. den persönlichen Lebenserfahrungen der Befragten haben (1-5). Die Antworten auf diese Fragen konnten ohne Schwierigkeiten und langes Nachdenken gegeben werden. Der Übergang zur Hauptphase, in der die , in Leitfäden skizzierten Themen- oder Fragekomplexe behandelt" (Reinders et al. 2011: 91) werden, fand beim ersten Themenkomplex statt, dabei ging es um die subjektive Auffassung der Migrationsliteratur und ihrer Position im Fremdsprachenunterricht (6-9). In der gleichen Phase wurde der zweite Themenkomplex „Kulturelles Lernen“ besprochen (10-17).

Zum ersten Themenkomplex wurden die Befragten aufgefordert, Migrationsliteratur beschreibend zu definieren (7), ihre Rolle im Fremdsprachenunterricht einzuschätzen (8), sowie die Bedeutung historischer Kontexte für das Verstehen der Texte der Migrationsliteratur zu thematisieren (9). Der zweite Themenkomplex wurde durch die Aufforderung, kulturelles Lernen zu definieren (10), seine Bedeutung für die gegenwärtige Gesellschaft zu bestimmen (11) und im Rahmen des Fremdsprachenunterrichts zu relativieren (12), erfasst. Die Frage nach der Relevanz literarischer Texte der Migrationsliteratur für kulturelles Lernen (13) sollte die zwei Themenbereiche zusammenschließen. Mit Rückgriff auf einen 
selbst aufbereiteten Text wurden die Merkmale inter-/transkultureller Literatur abgefragt (14). Auf die Herausforderungen und Bereicherungen der kulturellen Texte gehen die Fragen 15 und 16 ein. Die letzte Frage kennzeichnet zum einen das Ende des Interviews und gibt zum anderen die Möglichkeit, Unangesprochenes in Bezug auf die Themenkomplexe ins Gespräch zu bringen (17).

Als kritische Reflexion der Methode sei einschränkend gesagt, dass lediglich die deklarativen Konzepte des kulturellen Lernens an russischer Migrationsliteratur der LehramtsanwärterInnen erhoben wurden, was ausschließlich deren Aussagen über ihr Verständnis der Themenbereiche umfasst. Keinesfalls wurden die Interviews zum Überprüfen des in der Lehrveranstaltung vermittelten Wissens eingesetzt. Primär ging es um die individuellen Kognitionen und Argumentationen der Studierenden und damit um den Lernprozess im konstruktivistischen Sinne. Denn diesem zufolge ist Lernen ,ein aktiver Prozess der Wissenskonstruktion, bei dem Informationen aufgenommen und interpretiert werden und sich dann als individuell repräsentiertes Konstrukt [...] manifestieren“ (Haß 2017: 225). Die subjektiven Interpretationen der angehenden LehrerInnen sind insofern für die Erforschung der Lernprozesse in der Lehramtsausbildung von Bedeutung, als sie potenzielle Einsätze der kulturellen Thematik in ihrer beruflichen Zukunft vorzeichnen. Nicht zufällig betont Hallet den Einfluss subjektiver Annahmen von Studierenden: „Die in der wissenschaftlichen Ausbildung entwickelten und vermittelten Kompetenzen sind nur dann bildungswirksam, wenn sie auch aus Sicht der Studierenden mit den in der außeruniversitären Wirklichkeit erforderten Kenntnissen und Fähigkeiten kompatibel sind“" (Hallet 2013: 22).

An der Studie nahmen sieben Studierende teil. Sie sind zwischen 24 und 37 Jahre alt, fünf Frauen und zwei Männer. Sie kommen aus Österreich (3), Russland (1), der Ukraine (1), Spanien (1) und Großbritannien (1). Alle TeilnehmerInnen haben im Laufe ihrer Ausbildung Kontakt mit min-

Anastasia Drackert und Katrin Bente Karl (Hg.), Didaktik der slawischen Sprachen

Beiträge zum 2. Arbeitskreis in Innsbruck (19.02.-20.02.2018)

(C) 2019 innsbruck university press, ISBN 978-3-903187-80-1, DOI 10.15203/3187-80-1 
destens drei Fremdsprachen. Die Gruppe ist heterogen hinsichtlich des Niveaus der Sprachbeherrschung (ein Muttersprachler, ein Herkunftssprecher, zwei Studierende B2, drei Studierende B1) sowie der Semesterzahlen $(9,7,10,9,11,12,7)$. Wegen der hohen Heterogenität der Gruppe wurden die Texte auf Russisch und Deutsch angeboten, was zum besseren Verständnis der sprachlichen und emotionalen Tiefe des Erzählten führte.

\subsection{Forschungsergebnisse}

Es ging in der Studie um die mentalen Bilder der angehenden LehrerInnen hinsichtlich zweier Bereiche: des Einsatzes russischer Migrationsliteratur im Kontext kulturellen Lernens und des Verständnisses kulturellen Lernens im Fremdsprachenunterricht.

Der Einfluss der (Lern-)Biografien der Befragten auf die Wahrnehmung des Themas zeigt sich zunächst in ihrer Auffassung des eigenen Bezugs zur Migration. Alle Befragten weisen Erfahrungen mit Migration auf, dabei erwähnen zwei Personen (S3, S5) die Abhaltung von DaF-Kursen für MigrantInnen, ein Proband (S1) verbindet den Begriff mit einer hohen Anzahl von MigrantInnen in Österreich im Allgemeinen, vier Personen (S2, S4, S5, S6) gehen auf biografische Details der eigenen Migrationsgeschichte ein, ein Proband (S7) nennt in diesem Zusammenhang Vorurteile gegenüber und Mitleid mit MigrantInnen.

Die Vorerfahrungen mit kulturellem Lernen beziehen sich auf die Lehrveranstaltungen der universitären Ausbildung (S1, S2, S3, S4, S5, S7) und negative Vorfälle in Bezug auf Bewerbungen im Berufsleben (S6). Auf die Aufforderung, Texte der Migrationsliteratur zu nennen, die sie vor der Lehrveranstaltung gekannt haben, erwähnen zwei der Befragten (S1, S5) 
Wolfgang Herrndorfs Roman „Tschik“, der eine fachdidaktische Reflexion in der Germanistik findet (vgl. Ivanova 2016), sowie den Roman „Löcher" von Lois Sachar. Ein Proband (S2) erinnert sich an das Buch von Sam Salvin „Londonnes“; drei Probanden (S3, S4, S7) geben an, dass sie keine Texte der Migrationsliteratur zuvor gelesen haben; eine Person (S6) erinnert sich an einen Text von Nabokov.

In der subjektiven Auslegung der Migrationsliteratur als Gattung sind sich die ProbandInnen relativ einig, Migration oder Migranten müssen in der Handlung vorkommen (S1, S2, S4, S5) oder der Autor muss einen Migrationshintergrund haben (S5, S7). Ein Befragter (S3) erklärt Migrationsliteratur sehr umfassend: „Das sind einfach Texte, die sich mit verschiedenen Kulturen befassen, oder auch mit Fremdheit, Identität, einfach die, die die Verschiedenheit und Fremdheit darstellen, es muss nicht unbedingt sein, dass irgendwer auswandert oder in ein anderes Land kommt. [...] Andersartigkeit im eigenen Land kann auch thematisiert werden." Solch eine breite Auffassung kann aus der simultanen Behandlung der Migrationsthematik mit kulturellem Lernen resultieren.

Die Bedeutung der Migrationsliteratur für den Fremdsprachenunterricht wird facettenreich erklärt. Angefangen von der allgemeinen Auffassung, dass diese Texte ,zentraler Thematik“ (S1) und „sehr bedeutend“ (S2) sind, konkretisieren die Befragten den Stellenwert dieser Literatur als Mittel zur Vermittlung von Informationen über Sehenswürdigkeiten, Straßen, Orte, unbekannte Besonderheiten (S5), des Weltwissens und der Weltkultur (S2, S4, S7), zur Relativierung der eigenen (Familien-)Geschichte (S2) und der Geschichte der Heimat (S4), zur Reflexion der zeitgenössischen Diskurse sowie zur Herstellung einer Verbindung zwischen den historischen und den gegenwärtigen Diskursen (S3). Darüber hinaus veranschaulicht die Migrationsliteratur Menschen und ihre Gefühle (S4) und bietet Anlass zum Nachdenken (S5). Die wenigen Kritikpunkte beziehen sich auf den nicht kanonischen Charakter der Migrationsliteratur (S1), was ihren

Anastasia Drackert und Katrin Bente Karl (Hg.), Didaktik der slawischen Sprachen

Beiträge zum 2. Arbeitskreis in Innsbruck (19.02.-20.02.2018)

(C) 2019 innsbruck university press, ISBN 978-3-903187-80-1, DOI 10.15203/3187-80-1 
Einsatz im Unterricht erschwert, sowie auf die Gefahr einer stereotypen Wahrnehmung anderer Kulturen (S4, S7).

Der Einfluss der historischen Kontexte auf das Verstehen der Texte russischer Migrationsliteratur wird in der Aussage eines der Probanden (S1) repräsentativ für andere Teilnehmer hervorgehoben: „Einen russischen Text zur Migrationsliteratur zu lesen ohne irgendwie die Ereignisse von 1917 und 1945, ohne die sozialistische Wende von 1989-90 zu besprechen, hat wenig Sinn." Durch die Kenntnis der Geschichte kann man das Verstehen des Erzählten erhöhen und eine eigene Meinung bilden (S1, S2, S3, S4, S5). Während ein Proband mit muttersprachlichen Russischkenntnissen eine kleine Auffrischung des Geschichtlichen bei der Arbeit mit der Migrationsliteratur für ausreichend hält (S6), erklärt ein anderer (S7), dass er vor dem Studium kein Wissen über die russische Revolution besaß und den Begriff Revolution ausschließlich mit Frankreich verband.

Kulturelles Lernen wurde von den Studierenden wie folgt aufgefasst: Die ProbandInnen S1 und S3 betonten, dass sie selbst als SchülerInnen kulturelles Lernen ausschließlich in Bezug auf landeskundliche Inhalte erlebten, was sich auf die Geschichte, Speisen, Begrüßungen, Gepflogenheiten, Schuluniformen und Weihnachten bezog, ,das Große, darauf bezogene hat es eigentlich bei uns nicht gegeben“ (S1). Unter dem „Großen“ wurden kulturelle Konzepte gemeint, die in der Schulausbildung dieser LehramtsanwärterInnen außer Acht gelassen wurden. Dennoch nennen ausnahmslos alle ProbandInnen an der ersten Stelle Kenntnisse über die fremde Kultur, die sich auf Sitten und Traditionen, den Alltag, Personen, Geschichte, Tischkultur sowie Begrüßungen, Bräuche und Sprichwörter beziehen. Erst danach erwähnen die ProbandInnen andere Ziele kulturellen Lernens, wie zum Beispiel die eigene vertraute kulturelle Situation zu relativieren, den Weltblick zu erweitern (S2), andere Kulturen kennen und respektieren zu lernen (S4), statt Differenzen zwischen den Kulturen Gemeinsamkeiten zu finden (S5, S6), über Traditionen und Bräuche zur Mentalität zu kommen und diese zu verstehen (S7). 
Die Bedeutung der Migrationsliteratur für kulturelles Lernen wurde allgemein als ,sehr wichtig“ (S6) eingeschätzt, konkret lässt sie sich durch die folgenden Aspekte zusammenfassen. Migrationsliteratur gibt Einblicke in die Gründe für Migration (S1) bzw. in die Autorensicht auf die Situation, die als Denkanstoß für SchülerInnen dienen kann, um eigene Positionen feststellen zu können (S3). Migrationsliteratur ist eng mit der Globalisierung Europas verbunden und hilft, Verständnis für andere aufzubauen (S4, S7). Texte der Migrationsliteratur verbinden die kulturelle Thematik mit der Vermittlung von Lexik und Grammatik (S5).

Das Verständnis der Unterschiede zwischen inter- und transkulturellen Texten ist bei den ProbandInnen nicht deutlich. Sie zeigen Unsicherheit bei der Aufforderung, einen der gelesenen Texte als inter- oder transkulturell zu bezeichnen (S1, S2, S3, S4, S6). Dabei versuchen sie diese Unsicherheit zu begründen: „Trans- ist zwischen Kulturen und inter- ist auch zwischen Kulturen“(S5), ,bei der trans- da wird es schon recht vermischt, aber hier ist es schon recht getrennt, das ist interkulturell“ (S3), ,interkulturell - man bekommt viele Infos über Leute und den Lebensmodus der Leute“ (S4). Spannend ist die unerwartete Diskrepanz in der Wahrnehmung des Textes von Kaminer. Während Proband S3 „Russendisko“ wie folgt beschreibt: „Das ist schon recht interkulturell, es gibt die Kultur und auf der anderen Seite die andere Kultur, dass man so viel mit den Stereotypen richtig spielt", argumentiert Proband S7 mit der Beschreibung des Protagonisten für die transkulturelle Ausrichtung des Textes: „Und man hat von ihm gar keine Identitätskrise bemerkt, also bei ihm war da irgendwie kein Konflikt zwischen seiner Identität und der neuen Umgebung. Er war sich seiner Identität bewusst, und er hat sich mehr als zurechtgefunden in der neuen Umgebung“" (S7).

Hinsichtlich der Schwierigkeiten bzw. Herausforderungen bei der Arbeit mit Texten der Migrationsliteratur heben die Probanden sehr unterschiedliche Aspekte hervor. Es geht um die persönliche Distanzierung bei jenen Texten, die an die eigene familiäre Situation bzw. das Erlebte erinnern (S2)

Anastasia Drackert und Katrin Bente Karl (Hg.), Didaktik der slawischen Sprachen

Beiträge zum 2. Arbeitskreis in Innsbruck (19.02.-20.02.2018)

(C) 2019 innsbruck university press, ISBN 978-3-903187-80-1, DOI 10.15203/3187-80-1 
sowie um die herausfordernde Lexik, die sowohl nach stilistischen als auch thematischen Zusatzerklärungen verlangt (S5, S7). Es betrifft den geschichtlichen Hintergrund des Erzählten, wobei wiederum auf die Differenzen zwischen den MuttersprachlerInnen und RussischlernerInnen hingewiesen wird (S6, S7). Ein Proband (S3) betont die Bedeutung der Abgrenzung zwischen den wahren geschichtlichen Ereignissen und einer fiktiven Erzählung bzw. Perspektive, die im literarischen Text angeboten wird: „Dass man den historischen Kontext verstehen muss, auch die verschiedenen Ansichten herausfiltern muss, die Ansicht des Autoren, vielleicht von der historischen Sicht, was ist vielleicht fiktiv darin, was man vielleicht dazu filtern kann, und auch was ist ein Stereotyp, und was ist wirklich so."

Auf die Frage der persönlichen Relevanz bzw. möglichen Implikationen für ihr weiteres (Berufs-)Leben nach der Auseinandersetzung mit kulturellen Texten der Migrationsliteratur antworteten die Probanden relativ unpersönlich und allgemein. Wegen der Immanenz des Themas in unserer Gesellschaft würde man diese Lektüre in den eigenen Unterricht mit einbeziehen (S1). Literatur an sich soll im Fremdsprachenunterricht einen verdienten Platz bekommen (S2) und für die Spracharbeit hinsichtlich der Lexik und Grammatik eingesetzt werden (S5). Man würde die Arbeitsweise bzw. den Umgang mit sowie die Fragestellungen zu den Texten der Migrationsliteratur aus der Lehrveranstaltung in den Unterricht übernehmen (S3). Man würde das Niveau der Sprachbeherrschung und das Alter der SchülerInnen (S4) besser berücksichtigen sowie das Vorwissen der LernerInnen in die kulturellen Vergleiche (S5) bei der Arbeit mit diesen Texten mit einbeziehen. Ein Proband sieht als Konsequenz aus dieser Lehrveranstaltung einen starken Impuls zur stetigen Weiterbildung hinsichtlich des geschichtlichen Wissens über Russland (S7). 


\subsection{Diskussion der Ergebnisse}

Das Ziel dieser Studie war, die Aussagen der Lehramtsstudierenden nicht verallgemeinernd $\mathrm{zu}$ erfassen, sondern die breite Palette ihrer Ansichten und Wahrnehmungen hinsichtlich des Einsatzes russischer Migrationsliteratur im kulturellen Lernen festzuhalten. Dennoch lassen sich gewisse Ähnlichkeiten bzw. Unterschiede feststellen. Auffallend waren deutliche Schwierigkeiten der Studierenden, ihre Erfahrungen aus der Lehrveranstaltung zu reflektieren, was als mangelnde Diskursfähigkeit interpretiert werden kann. Die Fähigkeit, an der fachlichen und außerfachlichen Kommunikation in Bezug auf das Thema teilzunehmen, stellt aber eine der wichtigsten Komponenten des kompetenzentwickelnden Hochschulunterrichts (vgl. Hallet 2013: 19) dar. Als Konsequenz daraus soll in der folgenden Lehre eine verstärkte Förderung der Diskurskompetenz angeboten werden. Die anderen beiden Prozesse, nämlich Kognition und Interaktion, sind bei der schriftlichen Klausur und den Unterrichtssimulationen der Studierenden deutlich zum Vorschein gekommen.

Aus der Sicht der Studierenden eignet sich die Migrationsliteratur bestens zum Einsatz im kulturellen Lernen. Ausschließlich positive Reflexionen bezeugen ihre Bedeutung in der Ausbildung angehender RussischlehrerInnen.

Die Fragen zur Wahrnehmung des kulturellen Lernens im Unterricht sowie zu persönlichen Implikationen geben Einsichten in die mentalen Konstruktionen, die für die Reflexion der kulturellen Ziele von großer Bedeutung sind. Demnach versteht man unter kulturellem Lernen eine enge Kombination aus landeskundlichem Wissen und den in der Identitätskompetenz verorteten kulturellen Zielen. Das könnte auf das Erreichen der kulturellen Ziele im Rahmen dieser Lehrveranstaltung hinweisen, jedoch deuten die konkreten Ausprägungen möglicher Implikationen für das weitere Berufs-

Anastasia Drackert und Katrin Bente Karl (Hg.), Didaktik der slawischen Sprachen

Beiträge zum 2. Arbeitskreis in Innsbruck (19.02.-20.02.2018)

(C) 2019 innsbruck university press, ISBN 978-3-903187-80-1, DOI 10.15203/3187-80-1 
leben auf eine geringe Bedeutung des „großen Kulturellen“ hin. Der Aufbau kultureller Kompetenzen verläuft sehr individuell und ist durch Persönlichkeitsmerkmale und Lebens-/Lernerfahrungen geprägt. Aus diesem Grund kann die Tiefe der Reflexionsergebnisse eher kritisch wahrgenommen werden, was zur Folge hat, dass die kulturelle Kompetenz nicht deutlich gemessen werden kann.

\section{Fazit}

Der Beitrag stellt ein Plädoyer für den Einsatz der Migrationsliteratur im kulturellen Lernen dar. Anhand von Migrationsliteratur wurde versucht, sowohl kognitive als auch affektive und persönlichkeitsbezogene Prozesse im Unterricht zu initiieren. Die aktive Teilnahme sowie die durchaus positiven Rückmeldungen der Studierenden zur Lehrveranstaltung verdeutlichen das große Potenzial der Migrationsliteratur für das kulturelle Lernen im Lehramtsstudium. Die Studie zeigt eine Möglichkeit, kulturelles Lernen zu reflektieren, verdeutlicht aber zugleich die Herausforderungen und mögliche Grenzen dieser Reflexion.

\section{Literatur}

Alter, G. (2016). Transkulturelle Kompetenzen durch transkulturelle Literatur - Implikationen für den Englischunterricht. In M. Rückl (Hrsg.), Sprachen \& Kulturen. Vermitteln und vernetzen (50-61). Münster: Waxmann.

Altmayer, K. (2009). Instrumente für die empirische Erforschung kultureller Lernprozesse im Kontext von Deutsch als Fremdsprache. In A. 
$\mathrm{Hu} \&$ M. Byram (Hrsg.), Interkulturelle Kompetenz und fremdsprachliches Lernen. Modelle, Empirie, Evaluation (123-138). Tübingen: Gunter Narr Verlag.

Bloom, B. (Hrsg.). (1986). Taxonomie von Lernzielen im kognitiven Bereich. Weinhelm, Basel: Belz Verlag.

Bock, M. (1991). Das halbstrukturierte-leitfadenorientierte Tiefeninterview. In J. H. Hoffmeyer-Zlotnik (Hrsg.), Analyse verbaler Daten (90-109). München: Psychologie Verlagsunion.

Bredella, L. \& Christ, H. (Hrsg.). (1995). Didaktik des Fremdverstehens. Tübingen: Gunter Narr Verlag.

Bredella, L. (2017). Literaturdidaktik. In C. Surkamp (Hrsg.), Metzler Lexikon Fremdsprachendidaktik (2. Aufl.) (233-237). Stuttgart: Metzler Verlag.

Bruner, J. (1986). Actual Minds, Possible Worlds. Harvard: Harvard UP. Byram, M. (1997). Teaching and Assessing Intercultural Communicative Competence. Clevedon: Multicultural Matters.

Caspari, D. \& Küster L. (Hrsg.). (2010). Wege zu interkultureller Kompetenz. Fremdsprachendidaktische Aspekte der Text- und Medienarbeit. Frankfurt am Main: Peter Lang Verlag.

Csorba, A., Grunda, M., Gürtler, C., Hargaßner, J., Hausbacher. E., Horvath, A., Pabis, E. \& Trippo, S. (Hrsg.). (2018). Grenzgängerinnen: Migrationsgeschichten in der Gegenwartsliteratur. Ein kulturwissenschaftliches Studienbuch. Wien: Praesens Verlag.

Delanoy, W. (2014). Transkulturalität als begriffliche und konzeptuelle Herausforderung an die Fremdsprachendidaktik. In F. Matz, M. Rogge \& P. Siepmann (Hrsg.), Transkulturelles Lernen im Fremdsprachenunterricht. Theorie und Praxis (19-36). Frankfurt am Main: Peter Lang Verlag.

Fäcke, C. (2006). Transkulturalität und fremdsprachliche Literatur. Eine empirische Studie zu mentalen Prozessen von primär mono- oder bikulturell sozialisierten Jugendlichen. Frankfurt am Main: Peter Lang. 
Frederking, V. (Hrsg.). (2008). Schwer messbare Kompetenzen. Herausforderungen für die empirische Fachdidaktik. Baltmannsweiler: Schneider Verlag Hohengehren.

Freitag-Hild, B. (2010). Theorie, Aufgabenbeispiele und Unterrichtspraxis inter- und transkultureller Literaturdidaktik: British Fictions of Migration im Fremdsprachenunterricht. Trier: WVT.

Freitag-Hild, B. (2017). Interkulturelle kommunikative Kompetenz. In C. Surkamp (Hrsg.), Metzler Lexikon Fremdsprachendidaktik (2. Aufl.) (147-149). Stuttgart: Metzler Verlag.

Hallet, W. (2002). Fremdspracheunterricht als Spiel der Texte und Kulturen. Intertextualität als Paradigma einer kulturwissenschaftlichen Didaktik. Trier: WVT.

Hallet, W. (2007). Literatur und Kultur im Unterricht. Ein kulturwissenschaftlicher didaktischer Ansatz. In W. Hallet \& A. Nünning (Hrsg.), Neue Ansätze und Konzepte der Literatur- und Kulturdidaktik (3147). Trier: WVT.

Hallet, W. (Hrsg.). (2013). Literatur- und kulturwissenschaftliche Hochschuldidaktik. Konzepte, Methoden, Lehrbeispiele. Trier: WVT Wissenschaftlicher Verlag.

Haß, F. (2017). Lerntheorien. In C. Surkamp (Hrsg.), Metzler Lexikon Fremdsprachendidaktik (2. Aufl.) (224-226). Stuttgart: Metzler Verlag.

Hausbacher, E. (2009). Poetik der Migration. Transnationale Schreibweisen in der zeitgenössischen russischen Literatur. Tübingen: Stauffenberg Verlag.

Herder, J. G. (1774, 1990). Auch eine Philosophie der Geschichte zur Bildung der Menschheit. Stuttgart: Reclam Verlag.

$\mathrm{Hu}$, A. \& Byram, M. (Hrsg.). (2009). Interkulturelle Kompetenz und fremdsprachliches lernen. Modelle, Empirie, Evaluation. Intercultural competence and foreign language learning. Models, empiricism, assessment. Tübingen: Gunter Narr Verlag.

Ivanova, R. (2016). Gleich im Anders-Sein. Initiierung von inter- und transkulturellen Lernprozessen durch kooperative Lernformen. In

Anastasia Drackert und Katrin Bente Karl (Hg.), Didaktik der slawischen Sprachen

Beiträge zum 2. Arbeitskreis in Innsbruck (19.02.-20.02.2018)

(C) 2019 innsbruck university press, ISBN 978-3-903187-80-1, DOI 10.15203/3187-80-1 
M. Rückl (Hrsg.), Sprachen \& Kulturen. Vermitteln und vernetzen (62-72). Münster: Waxmann.

Kaes, A. (1990). New Historicism: Literaturgeschichte im Zeichen der Postmoderne? In H. Eggert, U. Profitlich \& K. R. Scherpe (Hrsg.), Geschichte als Literatur. Formen, Grenzen der Repräsentation von Vergangenheit (55-66). Stuttgart: Metzler.

Kramsch, C. (1993). Context and Culture in Language Teaching. Oxford: Oxford University Press.

Küster, L. (2010). Die Arte-Sendereihe „Karambolage“ - ein Anlass interkulturellen Lernens im Französischunterricht? In D. Caspari \& L. Küster (Hrsg.), Wege zu interkultureller Kompetenz (39-50). Frankfurt am Main: Peter Lang Verlag.

Küster, L. (2015). Warum ästhetisch-literarisches lernen im Fremdsprachenunterricht? Ausgewählte theoretische Fundierungen. In L. Küster, C. Lütge \& K. Wieland (Hrsg.), Literarisch-ästhetisches Lernen im Fremdsprachenunterricht. Theorie - Empirie - Unterrichtsperspektiven (15-32). Frankfurt am Main: Peter Lang Verlag.

O. A. (2016). Mitteilungsblatt 191 - Sondernummer der Paris Lodron-Universität Salzburg. Curriculum für das BA-Studium Lehramt der Universität Salzburg. (Allgemeinbildung) (Version 2016). Verfügbar unter: https://tinyurl.com/uzymjp3 [03.01.2019].

Reinders, H., Ditton, H., Gräsel, C. \& Gniewosz, B. (Hrsg.). (2011). Empirische Bildungsforschung. Strukturen und Methoden. Wiesbaden: VS Verlag für Sozialwissenschaften.

Riemer, C. (2016). Befragung. In D. Caspari, F. Klippel, M. K. Legutke \& K. Schramm (Hrsg.), Forschungsmethoden in der Fremdsprachendidaktik. Ein Handbuch (155-172). Tübingen: Narr Franke Attento Verlag.

Schulz, R. (2007). The challenge of assessing cultural understanding in the course of foreign language instruction. Foreign Language Annals 40(1), 9-26.

Welsch, W. (2012). Transkulturalität. In M. Kirloskar-Steinbach, G. Dharampal-Frick \& M. Friele (Hrsg.), Die Interkulturalitätsdebatte

Anastasia Drackert und Katrin Bente Karl (Hg.), Didaktik der slawischen Sprachen

Beiträge zum 2. Arbeitskreis in Innsbruck (19.02.-20.02.2018)

(C) 2019 innsbruck university press, ISBN 978-3-903187-80-1, DOI 10.15203/3187-80-1 
- Leit- und Streitbegriffe (146-156). München: Karl Aber.Primärliteratur (Original und Übersetzungen)

Бунин, И. (2008). В Париже /И. Бунин Темные аллеи (110-118). Москва: Профиздат.

Bunin, I. (2000). In Paris. In I. Bunin, Liebe und andere Unglücksfälle: Novellen (265-277). Frankfurt am Main: Eichborn Verlag.

Вертлиб, В. (2009). Остановки в пути. Санкт-Петербург: SYMPOSIUM.

Vertlib, V. (2003). Zwischenstationen. 2. Aufl. Wien, München: Franz Deuticke Verlagsgesellschaft.

Каминер, В. (2004). Russendisko. Москва: Новое литературное обозрение.

Kaminer, W. (2000). Russendisko. München: Wilhelm Goldmann Verlag. Кисина, Ю. (2012). Весна на луне. Санкт-Петербург: Азбука.

Kissina, J. (2013). Frühling auf dem Mond. Berlin: Surkamp Verlag.

Набоков, В. (1999). Пнин. Рассказы. Бледное пламя. СанктПетербург: Симпозиум.

Nabokov, V. (1994). Pnin. In V. Nabokov, Gesammelte Werke, Bd.9. 1. Aufl. Reinbek bei Hamburg: Rowohlt Verlag.

Рубина, Д. (2006). Вывеска /Итак, продолжаем (97-107). Москва: Эксмо.

Rubina, D. (2005). Ein Aushängeschild. In D. Rubina, Der letzte Eber aus den Wäldern Pontevedras. (267-274). Wien: Verlagshaus Pereprava.

Токарева, В. (2006). Своя правда /Можно и нельзя (7-80). Москва: Транзиткнига.

Tokarjewa; V. (2003). Eine Liebe fürs ganze Leben. Zürich: Diogenes Verlag. 